CASE REPORT

B.T. Yang
Z.C. Wang
J.F. Xian
O.H. Chen

\section{MR Imaging Features of Primary Mucosal Melanoma of the Eustachian Tube: Report of 2 Cases}

\begin{abstract}
SUMMARY: We observed 2 cases of malignant melanoma arising from the eustachian tube with MR imaging. In this region, we explored further characteristics of primary mucosal melanoma. We discuss the value of using MR imaging to diagnose and treat patients with melanoma in the eustachian tube. In addition, we introduce the key points to differentiate melanoma from some common diseases in the region.
\end{abstract}

M alignant melanoma primarily affects the skin, rarely the mucosa. Melanoma of the upper airway mucosa constitutes approximately $1 \%$ of the total melanoma of the head and neck. ${ }^{1}$ Primary melanoma of the eustachian tube is even rarer. In a series of 158 cases of mucosal melanoma of the upper airway, only 2 cases were located at the eustachian tube orifice in the nasopharynx. ${ }^{2}$ To the best of our knowledge, only 6 cases of primary melanoma of the eustachian tube have been reported in the literature to date $\mathrm{e}^{2-5}$; unfortunately, the corresponding MR imaging findings were not described effectively. During the past 5 years, melanoma of the eustachian tube in the nasopharynx has been positively proved through histopathology in 2 patients at our hospital. These 2 patients underwent MR imaging.

\section{Case Reports}

\section{Case 1}

A 75-year-old man with mild hypertension and an intermittent epistaxis for the past 20 days was examined. An otorhinolaryngologic examination showed serous otitis media of the left ear and a brownblack soft-tissue mass with surface irregularity in the left nasopharynx. The pharyngeal orifice of the left eustachian tube was obstructed. MR imaging revealed an elliptic well-defined $1.2 \times 2.0 \times 1.4 \mathrm{~cm}$ mass in the left nasopharynx, arising from the left eustachian tube. The lesion showed signal intensity hyperintense to gray matter on T1weighted images and hypointense signal intensity on T2-weighted images with moderate homogeneous contrast enhancement following the administration of contrast material (Fig $1 A-C$ ). The timeintensity curve of dynamic contrast-enhanced (DCE) MR imaging was a rapidly enhancing and rapid washout type (Fig $1 D,-E)$. The lesion was complicated with serous otitis media of the left ear, which showed hypointense signal intensity on T1-weighted images and hyperintense signal intensity on T2-weighted images without significant contrast enhancement. Neither an enlarged retropharyngeal node, evidence of head and neck melanoma, nor systemic abnormalities were observed. A surgical specimen of the brown-black mass in the left eustachian tube was histopathologically proved to be malignant melanoma. On immunohistochemistry, tumor cells were strongly positive for S-100, vimentin, and melanoma antibody HMB45.

Received June 19, 2008; accepted June 30

From the Department of Radiology, Beijing Tongren Hospital, Capital Medical University, Beijing, China.

Please address correspondence to Zhen Chang Wang, MD, Department of Radiology, Beijing Tongren Hospital, Capital Medical University, No.1, Dongjiaominxiang, Dongcheng District, Beijing 100730, China; e-mail:cjr.yangbentao@vip.163.com

DOI 10.3174/ajnr.A1270

\section{Case 2}

A 35-year-old woman with no significant medical history presented with a 2-year history of hearing decrease and with a 1-month intermittent epistaxis. An otorhinolaryngologic examination revealed serous otitis media of the left ear and an irregular coarse friable grayblack soft-tissue mass occupying the whole pharyngeal orifice of the left eustachian tube in the nasopharynx. MR imaging showed an elliptic-shaped well-defined $1.4 \times 2.5 \times 1.6 \mathrm{~cm}$ mass of the eustachian tube in the left nasopharynx, which was of low T2 and high T1 signal intensity with moderate homogeneous contrast enhancement (Fig 2). The lesion was associated with serous otitis media of the left ear, which showed hypointense signal intensity on T1-weighted images and hyperintense signal intensity on $\mathrm{T} 2$-weighted images without significant contrast enhancement following the administration of contrast material. Neither an enlarged retropharyngeal node, evidence of head and neck melanoma, nor systemic abnormalities were found. A biopsy specimen of the gray-black mass in the left eustachian tube revealed malignant melanoma. Immunohistochemically, the diagnosis was further confirmed by the expression of S-100 and melanoma antibody HMB45.

\section{Discussion}

Melanoma is composed of a proliferation of melanocytes, which derive from the neural crest and subsequently migrate into the nasal mucosal surfaces with an ectodermal origin. The etiology of mucosal melanoma is still unknown at present. ${ }^{6}$ The highest incidence occurs between the fifth and seventh decades of life, with a slight predilection in men. ${ }^{2}$ In the nasopharyngeal cavity, the most common clinical manifestations are intermittent epistaxis and hearing impairment.

MR imaging findings of the lesion largely depend on histologic features. ${ }^{7,8}$ Because of the paramagnetic characteristics of melanin, melanotic tumors may be detected by using MR imaging. Key observations are hyperintensity on T1-weighted and hypointensity on T2-weighted images. This paramagnetic property has been attributed to the free radical known to occur in melanin or in paramagnetic metals binding to melanin. The presence of this suggestive combination of signal intensities depends on the concentration and distribution of melanin in the tumor. The higher the melanin content of the tumor, the more intense is the signal intensity on T1-weighted and the lower is the signal intensity on T2-weighted images. ${ }^{7-12}$ As stated previously, our 2 patients showed similar MR imaging appearances, which indicated melanotic melanoma. Conversely, amelanotic melanoma will generally demonstrate hypointensity on T1-weighted and hyperintensity on T2- 

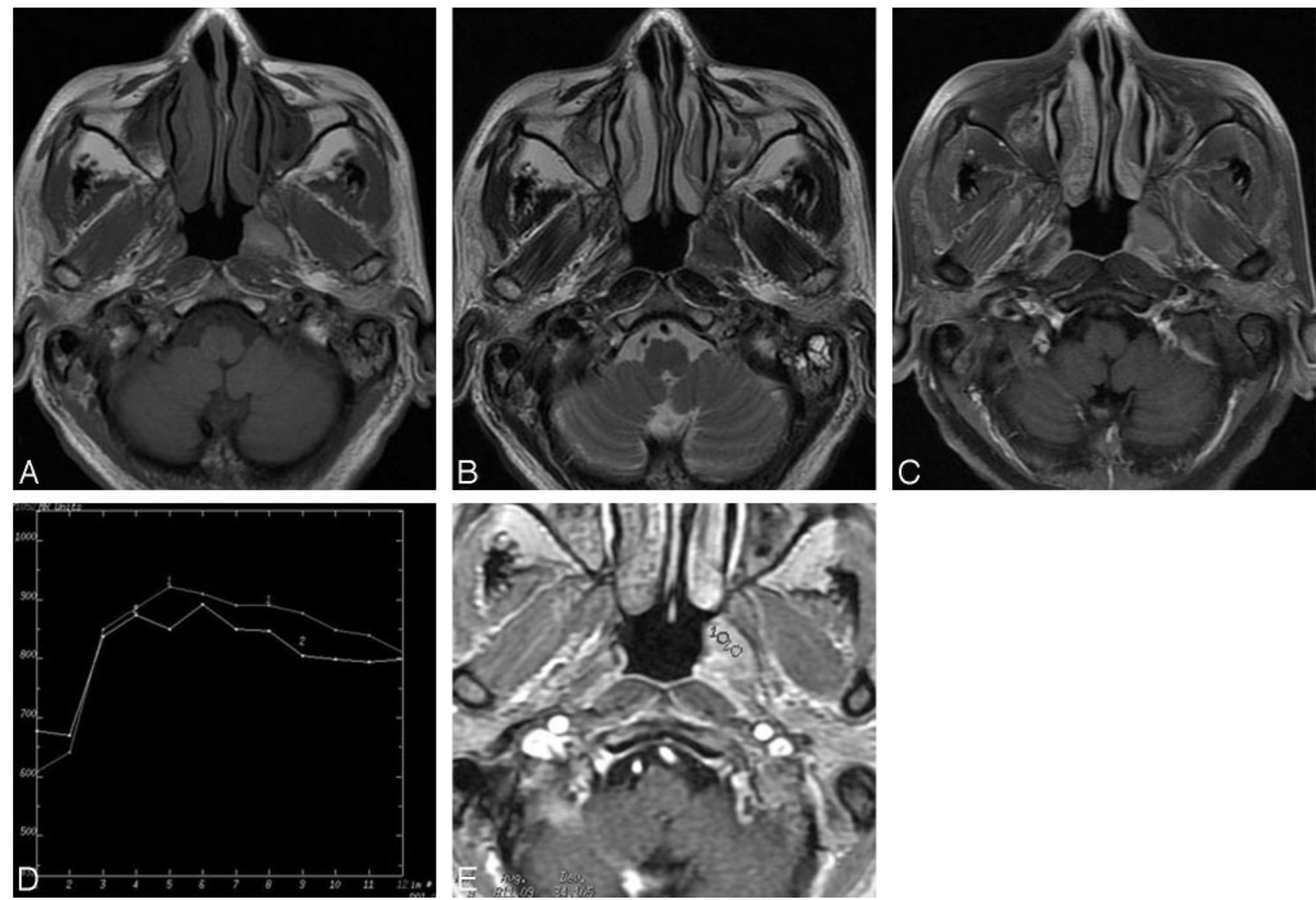

Fig 1. Case 1. A, Axial T1-weighted image shows a well-defined elliptic-shaped hyperintense-signal-intensity mass in the eustachian tube of the left nasopharynx $B$, The lesion has hypointense signal intensity on the axial T2-weighted image. C. Axial contrast-enhanced T1-weighted image with fat saturation shows moderate homogeneous enhancement of the lesion. $D$, Corresponding axial DCE MR image depicts the rapidly enhancing and rapid washout pattern. $E$, The 2 round cursors mark the region of interest selected for signal-intensity measurement at dynamic MR imaging.
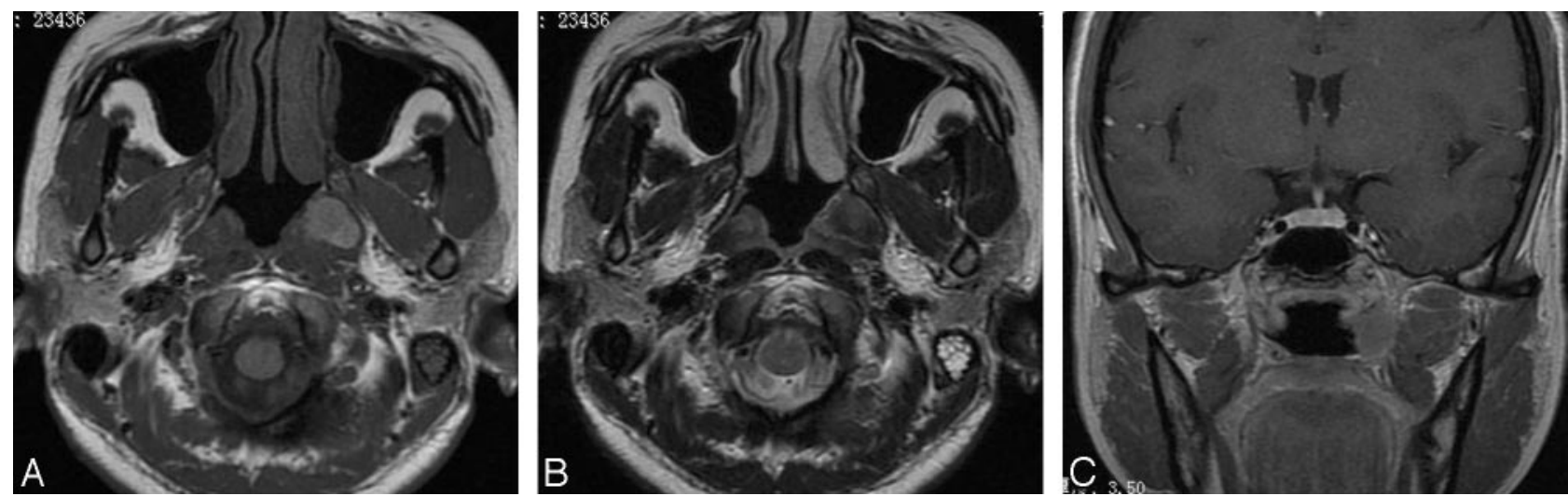

Fig 2. Case 2. A, Axial T1-weighted image shows a well-defined, elliptic-shaped hyperintense-signal-intensity mass in the eustachian tube of the left nasopharynx. B, The lesion has heterogeneous hypointense signal intensity on the axial T2-weighted image. $C$, Coronal contrast-enhanced T1-weighted image shows moderate enhancement of the lesion.

weighted images and thus is not easy to diagnose on the basis of the MR imaging alone. ${ }^{7,8}$

On the basis of these 2 cases, we believe that there are indications other than just signal intensity to aid in detecting melanoma of this region: brown- or gray-black color, ellipsis shape, and submucosal development toward the middle ear along the eustachian tube, accompanied by homolateral serous otitis media.

The lesion is difficult to diagnose by CT alone due to the inferior soft-tissue resolution of CT. In contrast, MR imaging has better soft-tissue resolution and is currently the best imaging technique for the evaluation of melanoma of the eustachian tube. MR imaging can clearly define the tumor and depict its site of origin. The typical appearance on MR imaging is strongly suggestive of the diagnosis for these lesions. MR imaging can accurately delineate the extent of the tumor, perineural spread, spread to adjacent structures, and accompanying changes and can provide more useful and reliable information for the treatment of choice for these patients.

The differential diagnoses for melanoma in the nasophar- 
ynx primarily include carcinoma, lymphoma, extramedullary plasmacytoma, and metastatic tumor. Carcinoma usually originates from the Rosenmüller fossa. Most lesions show intermediate signal intensity on T1-weighted MR images and slight hypointense signal intensity on T2-weighted images with associated bone destruction. Lymphoma is often bulky and homogeneous, revealing intermediate signal intensity on T1- and T2-weighted images, with moderate enhancement following the administration of contrast material. Extramedullary plasmacytoma is a rare soft-tissue malignancy composed of plasma cells. On T1-weighted MR images, the lesion may reveal a submucosal mass of low-to-intermediate signal intensity exhibiting moderate-to-marked contrast enhancement following the administration of contrast material. On T2-weighted images, it demonstrates intermediate-to-high signal intensity. Metastasis from hemorrhagic tumors and mucinous adenocarcinoma of the colon can appear as hypointense signal intensity lesions on T2-weighted MR images, though the absence of a primary tumor and the presence of homogeneous enhancement can also suggest the diagnosis of primary melanoma.

\section{Conclusions}

Although primary mucosal melanoma is extremely rare in the head and neck, it can occasionally arise in the eustachian tube. The signal-intensity features and enhancement patterns on
MR images may look similar to those of melanoma in other parts of the body and may help to support this diagnosis.

\section{References}

1. Urpegui García A, Lahoz Zamarro T, Muniesa Soriano JA, et al. Malignant melanoma of the middle ear, a rare site [in Spanish]. Acta Otorrinolaringol Esp 1999;50:559-62

2. Batsakis JG, Regezi JA, Solomon AR, et al. The pathology of head and neck tumors: mucosal melanomas. Head Neck Surg 1982;4(pt 13):404-18

3. Racic G, Kurtovic D, Roje Z, et al. Primary mucosal melanoma of the eustachian tube. Eur Arch Otorhinolaryngol 2004;261:139-42. Epub 2003 Jul 23

4. Lai CC, Tsay SH, Ho CY. Malignant melanoma of the eustachian tube. J Laryngol Otol 2001;115:567-69

5. Tywończuk-Szulc M, Kibilda B. Malignant melanoma of the auditory tube. Otolaryngol Pol 2007;61:335-38

6. Snow GB, van der Waal I. Mucosal melanomas of the head and neck. Otolaryngol Clin North Am 1986;19:537-47

7. Kim SS, Han MH, Kim JE, et al. Malignant melanoma of the sinonasal cavity: explanation of magnetic resonance signal intensities with histopathologic characteristics. Am J Otolaryngol 2000;21:366-78

8. Isiklar L, Leedss NE, Fuller GN, et al. Intracranial metastatic melanoma: correlation between MR imaging characteristics and melanin content. AJR Am J Roentgenol 1995;165:1503-12

9. Batra K, Chhabra A, Rampure J, et al. CT and MRI appearances of primary sphenoid melanoma: a rare case. AJNR Am J Neuroradiol 2004;26:2642-44

10. Ramos R, Som PM, Solodnik P. Nasopharyngeal melanotic melanoma: MR characteristics. J Comput Assist Tomogr 1990;14:997-99

11. Hammersmith SM, Terk MR. Jeffrey PB, et al. Magnetic resonance imaging of nasopharyngeal and paranasal sinus melanoma. Magn Reson Imaging 1990;8:245-53

12. Genc E, Karaarslan E, Hanci D, et al. Radiology quiz case 1. Arch Otolaryngol Head Neck Surg 2005;131:1023-27 